\title{
TÉNACITÉ D'ACIERS DE CONSTRUCTION À DIFFÉRENTES TEMPÉRATURES ET VITESSE DE CHARGEMENT
}

\author{
J.R. Klepaczko et G. Pluvinage* \\ Université de Nantes, ENSM, 1 me de la Noë, 44072 Nantes Cedex, France \\ *Laboratoire de Fiabilité Mécanique, Université de Metz, Ile du Saulcy, \\ 57045 Metz Cedex, France
}

Résumé - L'influence de la température de la vitesse de chargement a été étudiée sur la ténacité $k_{l c}$ et la limite d'élasticité $\sigma_{y}$ pour l'acier de cuve A508C1. 3 ainsi que d'autres aciers.

A partir de l'hypothèse du temps critique $t_{c}$ ou du concept de la température modifiée $T^{*}$, on montre la possibilité de construire la courbe maîtresse

$$
\log k_{l c}=f\left(\log _{y}\right)
$$

utile pour le choix d'un matériau utilisé dans diverses conditions de température et vitesse de déformation.

\footnotetext{
Abstract - The influence of temperature and loading rate upon the fracture toughness and yield stress for some structurals steels and particularly pressure vessel steels A508Cl.3.

With the hypothesis of critical time $t_{c}$, and modified temperature $T^{*}$ concept, it had been shown the possibility of constructing a master plot

$\log K_{l c} v s . \log \sigma_{y}$ which can be used for material selection for different conditions of temperature and strain rate.
}

\section{I - INTRODUCTION}

La modélisation de la tenacité des matériaux a été, jusqu'à présent axée sur le cas des ruptures quasi-statiques /1-4/. Cette modélisation fait intervenir un critère local de rupture dans lequel les caractéristiques de résistance à la rupture sont fonction uniquement de la température. L'utilisation d'un paramètre introduisant simultanément la vitesse de déformation et la température, paramètre issu de la théorie de l'activation thermique du mécanisme de déformation plastique $15-6 /$, a permis de représenter l'évolution de la ténacité en fonction de toute condition de température et de vitesse de déformation. Très récemment klepaczko /7-8/ et Pluvinage et Marandet /9/ ont introduit une modélisation plus rigoureuse en utilisant un critère local de rupture incorporant la sensibilité à ces deux paramètres. Dans la présente étude une série d'expériences systématiques dans une large gamme de températures et de vitesses de déformation sont utilisées pour étudịer ce problème. Ces expériences conduisent à l'établissement pour chaque matériau 
de relations suivantes :

- la limite d'élasticité $\sigma_{. y}$ est étudiée en fonction de la température et la vitesse de déformation $\stackrel{\bullet}{\varepsilon}$ :

$$
\sigma_{y}=f_{1}(\dot{\varepsilon}, T)
$$

- la ténacité $k_{l c}$ est étudiée en fonction de la température et de la vitesse d'augmentation du facteur d'intensité de contrainte $\dot{k}$ :

$$
k_{1 c}=f_{2}(\dot{K}, T)
$$

Ne conservant que l'hypothèse d'une plastification en fond de fissure régie par un phénonème thermiquement activé les auteurs présentent une méthode simple pour estimer la valeur de la ténacité $k_{l c}$ dans n'importe quelle condition de température et de vitesse de chargement. En outre il est possible de l'utiliser pour prévoir le décalage de la température de transition de ténacité.

\section{II - FAITS ET ARGUMENTS EXPERIMENTAUX}

Un certain nombre de résultats expérimentaux sont maintenant disponibles dans ce domaine $/ 10-14 /$. Ils permettent de schématiser le comportement typique de la ténacité $K_{l c}$ en fonction de la température $T$ et de la vitesse d'augmentation du facteur d'intensité de contrainte $\dot{k}$ (figure la et $1 \mathrm{~b}$ ).

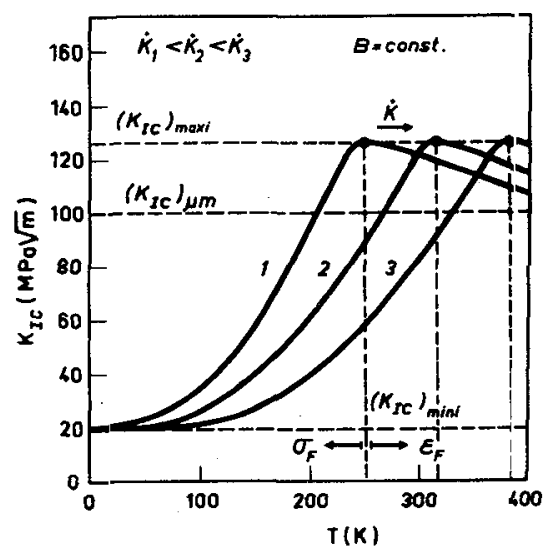

figure la

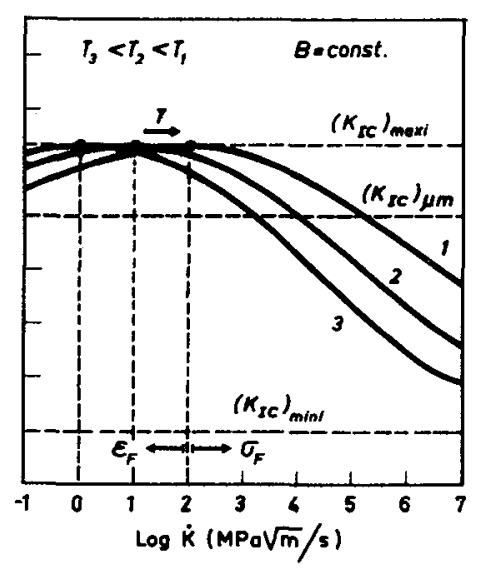

figure $1 b$

Figure 1 - Représentation schématique de la surface constitutive pour 3 températures et 3 valeurs de la vitesse d'augmentation du facteur d'intensité de contrainte.

- Schematic representation of the constitutive surface for proportional loadings, $k_{l c}=f_{2}\left(T, \dot{k}_{I}\right)$.

Selon les conditions, le processus de rupture d'un acier est controlé soit par le clivage, soit par la croissance et la coalescence de microvides. A basse température et à grande vitesse de déformation, la résistance à la rupture est fonction d'une contrainte critique de clivage (mode fragile). A haute température et à basse vites- 
se de déformation la ténacité dépend d'une déformation critique $\varepsilon_{F}$ (mode ductile). on remarque sur la figure $l$ que la surface constitutive $k_{l c}=f_{2}(T, \dot{K})$ est limitée par 2 plans, d'altitude $\left(K_{l c}\right)^{\prime}$ min et $\left(K_{l c}\right)_{\max }$ correspondant successivement au plateau fragile et au plateau ductile de ténacité des aciers.

Une modélisation réalisée par Klepaczko /8/ a permis de montrer que le plateau fragile correspond à une valeur caractéristique correspondant à la température de $0^{\circ} \mathrm{K}$. Cette valeur est une constante du matériau qui ne dépend uniquement que de la microstructure. Pour de nombreux aciers, cette valeur $\left(K_{l c}\right)_{\min }$ oscille autour de 20 MParm. Lorsque la température s'accroit, pour une valeur constante de $\dot{k}$, la ténacité $K_{1 c}$ augmente jusqu'au point de transition $(\dot{K}, T)_{c} \cdot$ La position de ce point de transition dépend de la température et de la vitesse de déformation. La valeur du maximum de ténacité $\left(K_{1 c}\right)_{\max }$ dépend non seulement de $\dot{k}$ et $T$, mais aussi de l'épaisseur du matériau, comme l'ont montré Krasovsky et AL /15/.

Lorsque la température dépasse le point de transition, la ténacité $k_{1 c}$ a tendance à décroitre et tend vers un autre niveau théorique $\left(K_{1 C}\right)_{\mu}$ qui peut lui aussi être prédit par une analyse basée conjointement sur un critère local de rupture, et une théorie de la plasticité activée thermiquement décrite dans $18 /$.

La figure lb montre que le plateau supérieur, obtenu à basse vitesse de changement présente une sensibilité positive à la vitesse de chargement, c'est-à-dire que la ténacité augmente lorsque la vitesse de chargement augmente. La partie correspondant au plateau fragile possède une sensibilité négative.

Le point de transition se déplace dans le même sens que la température et la vitesse de chargement. La modélisation a permis de montrer que la valeur ( $\left.K_{l c}\right)^{\prime}$ max augmente très légèrement avec la vitesse de déformation.

Remarquons que les schémas de la figure 1 reposent sur l'hypothèse que la ténacité $K_{l c}$ puisse être déterminée au niveau du plateau supérieur dans des conditions acceptables selon la norme ASTM E399, ou à défaut qu'une ténacité équivalente $K_{\text {Jc }}$ puisse être mesurée.

Des corrélations entre les caractéristiques de traction et la ténacité des matériaux ont été mises en évidence par différents auteurs Hahn et al. (16) à partir de résultats d'essais sur une dizaine d'aciers a trouvé une relation du type

$$
\sigma_{F / \sigma_{y}}=A_{2}\left(K_{l c} / \sigma_{y}\right)^{\beta}
$$

où $\sigma_{F}$ est la contrainte critique de clivage $A_{2}$ et $\beta$ les constantes. Une corrélation d'un type similaire a été proposée par Holtzmann et al /12/ à partir de résultats obtenus pour, deux vitesses de déformation. Dahl et al $/ 6 /$ ont indiqué une corrélation entre la ténacité $\kappa_{1 c}$ et la limite d'écoulement $\sigma_{3}$ définie pour une déformation $\varepsilon=0,03$.

Qualitativement ces corrélations montrent que lorsque la limite d'élasticité augmente, que ce soit sous l'effet de l'abaissement de la température d'essai ou de 1 'augmentation de la vitesse de déformation. la ténacité décroit. 


\section{III - RESULTATS EXPERIMENTAUX}

Afin d'obtenir de bonnes corrélations deux types d'essais ont été réalisés sur l'acier SA 508Cl.3. La limite d'élasticité a été déterminée dans une large gamme de température pour 4 vitesses de déformation :

$\dot{\varepsilon} \leqq 1,3 \times 10^{-3} \mathrm{~s}^{-1}$ à 1 'aide d'essais de traction (points ouverts) ou d'essais de compression (points noirs)

$\dot{\varepsilon}=0,4 \mathrm{~s}^{-1}$ à $l^{\prime}$ aide d'essai de traction (carré blanc)

$\dot{\varepsilon}=2 \mathrm{~s}^{-1}$ essais de compression

$\dot{\varepsilon}=2 \times 10^{3} \mathrm{~s}^{-1}$ par essais de compression réalisés sur barres d'Hopkinson.

On peut remarquer sur la figure 2 que la limite d'élasticité de l'acier SA $508 \mathrm{Cl} 3$ est sensible à la vitesse de déformation. Les différentes courbes convergent vers une valeur unique de la limite d'élasticité à OK ; pour les basses vitesses de déformation le plateau athermique $\sigma_{y \mu}$ est atteint.

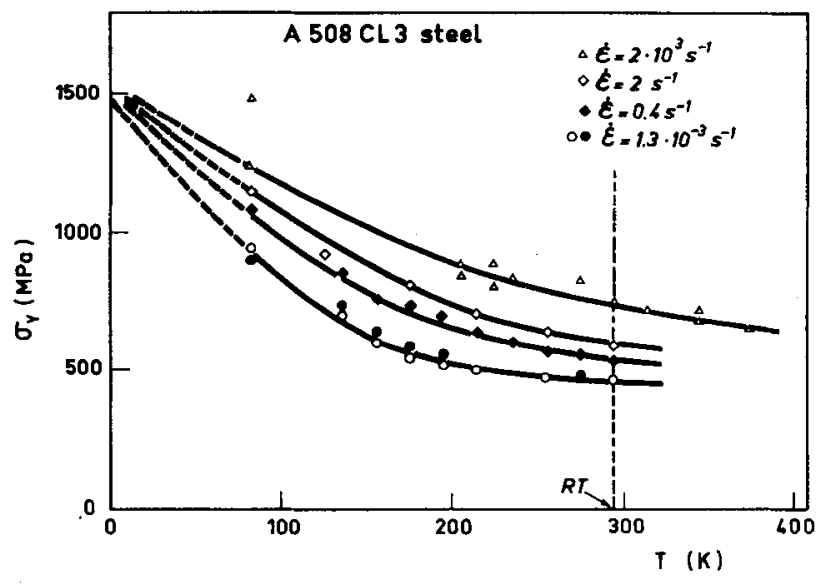

Figure 2 - Evolution de la limite d'élasticité en fonction de la température $T$ pour différentes vitesses de déformation ; Acier SA $508 \mathrm{Cl} .3$

Variation of yield stress $\sigma_{y}$ as a function of temperature $T$ for different strain rates; pressure vessel steel $\mathrm{A} 508 \mathrm{Cl} .3$.

La ténacité $K_{l c}$ a été déterminée pour le même acier et pour 4 vitesses différentes $d$ 'augmentation du facteur d'intensité de contrainte $\dot{k}$. Les points expérimentaux relatifs aux 3 vitesses de chargement les plus basses ont été réalisés à l'aide d'une technique expérimentale développée par Marandet et al /18/ à l'IRSID. Les points expérimentaux du plateau supérieur sont obtenus à partir de la valeur critique du paramètre energétique $J$.

Pour l'obtention de la ténacité à grande vitesse de chargement $\dot{\mathrm{K}} \simeq 10^{6} \mathrm{MPa} / \mathrm{m} / \mathrm{s}$, ont été utilisées des éprouvettes WLCT et une méthode de chargement par barres d'Hopkinson $/ 19 /$. 


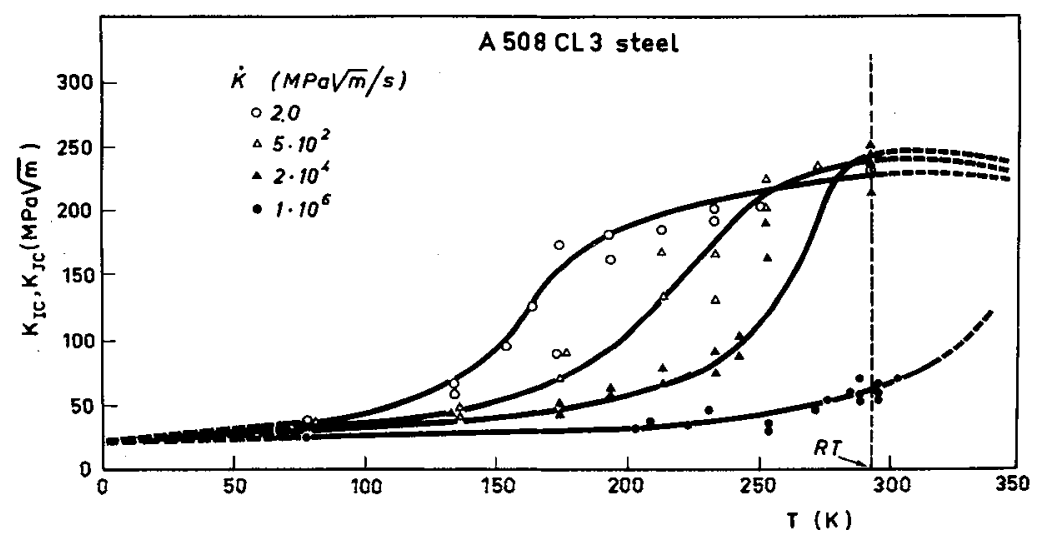

Figure 3 - Evolution de la ténacité $K_{l c}$ au $K_{J c}$ en fonction de la température $T$ pour 4 vitesses de chargement $\dot{k}$; Acier SA $508 \mathrm{Cl} 1.3$.

Variation of fracture toughness $K_{I C}$ or $K_{J C}$ as a function of temperature $T$ for four different loading rates $\dot{K}$; pressure vessel steel $A 508 \mathrm{Cl} .3$.

On constate que cet acier présente une ténacité très sensible à la vitesse de déformation, sensibilité négative. A titre d'exemple pour une augmentation de la vitesse de chargement de $2 \mathrm{MPa} / \mathrm{m} / \mathrm{s}$ à $1 \times 10^{6} \mathrm{MPa} / \mathrm{m} / \mathrm{s}$, le rapport des ténacités statiques et dynamiques $\xi=\left(K_{1 c}\right) /\left(K_{1 c}\right)_{D}$ prend une valeur moyenne de $\xi=5,5$ dans l'intervalle de température $175 \mathrm{~K} \leqq T \leqq 275 \mathrm{~K}$, la diminution la plus importante $\xi=$ $-5,8$ apparait à $T=210 \mathrm{~K}$. D'autres résultats similaires sur les aciers $\mathrm{XC} 38$, $\mathrm{XC2}$ et 35NCDV2 sont reportés dans /20/

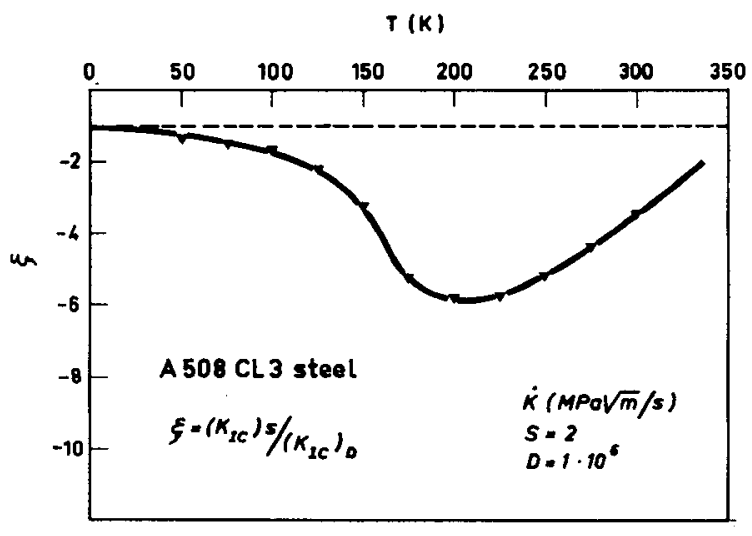

Figure 4 - Rapport de la ténacité $\xi$ en fonction de la température $T$ pour 2 vitesses de chargement $\dot{K}$ extrêmes ; Acier SA 508C1.3

Ratio of fracture toughness $\xi$ as a function of temperature $T$ for two extreme loading rates $\dot{K}$; pressure vessel steel $\mathrm{A} 508 \mathrm{Cl} .3$. 


\section{IV - CORRELATIONS}

Une corrélation entre l'évolution, en fonction de la vitesse de déformation et de la température, de la limite d'élasticité et de la ténacité peut être obtenue en utilisant le temps à rupture $t_{c}$. Celui-ci peut être relié à la vitesse de déformation $\dot{\varepsilon}$ et la vitesse de chargement $\dot{K}$ par :

$$
t_{c}=\frac{\varepsilon_{c}}{\dot{\varepsilon}} \quad \text { et } \quad t_{c}=\frac{K_{l c}}{k}
$$

En éliminant le temps entre (4) il vient :

$$
\dot{k}=\frac{k_{l c}}{\varepsilon_{c}} \quad \dot{\varepsilon}
$$

On considérera que le matériau possède un écrouissage linéaire de sorte que

$$
\sigma=\alpha E \varepsilon \quad \text { et } \quad E_{t}=\alpha E
$$

$\alpha$ est une constante et vaut $1 \leqq \alpha \leqq 0,05$ pour la plupart des aciers à faible taux de déformation de sorte que :

$$
\dot{K}=\alpha \frac{E K I c}{\sigma} \dot{\varepsilon}
$$

L'utilisation d'une échelle logarithmique conduit à :

$$
\log \dot{K}=\log \left(\frac{E K}{\sigma}\right)+\log \dot{\varepsilon}
$$

On considère la constante $c=\log \left(\alpha \frac{E K_{l c}}{\sigma}\right)$ appelée le facteur de conversion logarithmique $/ 8 /$; on a constaté que dans la plupart des cas $3 \leqq C \leqq 4$. L'expression (6) permet de choisir les couples de points $\kappa_{l c}$ et $\sigma_{y}$ ayant des vitesses équivalentes selon cette équation.

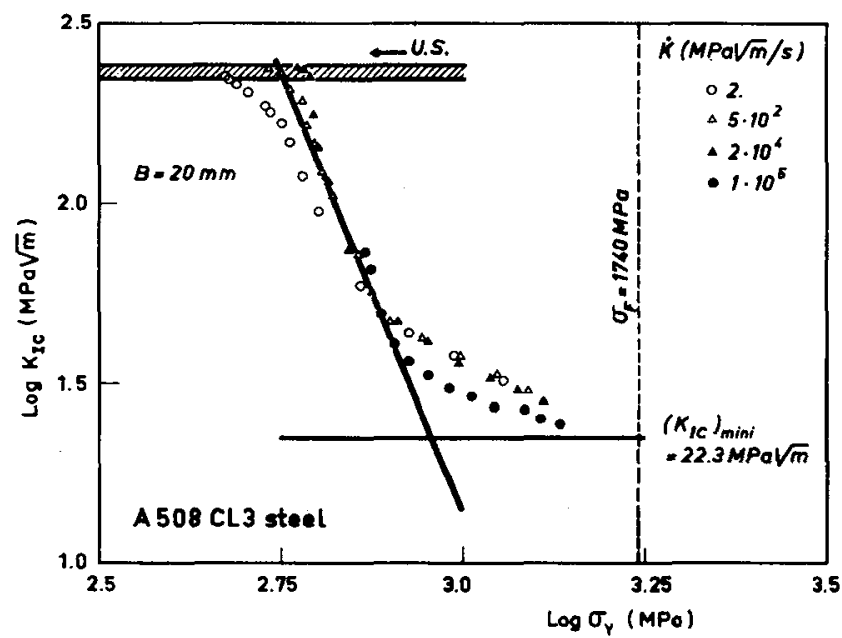

Figure 5 - Courbe maîtresse de l'acier SA 508C1.3 - épaisseur de l'échantillon $B=20 \mathrm{~mm}$ U.S. - plateau supérieur (upper shelf).

Master plot for $A 508$ steel, specimen thickness $B=20 \mathrm{~mm}$, US-upper shelf. 
Les 4 courbes $\sigma_{y}(T) \dot{\varepsilon}$ sont approximativement équivalentes selon l'expression (6) aux 4 courbes $K_{l c}(T)_{\dot{K}}$. Une courbe maitresse $\log k_{l c}$ en fonction de $\log \sigma_{y}$ peut être ainsi facilement tracée et présentée dans la figure 5. La valeur minimale de la ténacité, valeur caractéristique à $O K$ du matériau donc indépendante de la température et de la déformation est estimée comme étant égale à $\left(K_{1 c}\right)_{m i n}=22,3 \mathrm{MPa} / \mathrm{m}$. Entre cette valeur minimale et le plateau supérieur, la région de transition peut être approximée grossièrement par une droite de pente négative dans le diagramme bilogarithmique $\log K_{1 c}=f\left(\log \sigma_{y}\right)$. Ce diagramme est limité à droite par la verticale d'abscisse $\log \sigma_{F}$ où $\sigma_{F}$ est la contrainte critique de clivage de l'acier SA $508 \mathrm{Cl} .3, \sigma_{F}$ a été mesuré et est de l'ordre de $\sigma_{F}=1740 \mathrm{MPa}$.

Une telle corrélation a été aussi obtenu à partir des données expérimentales de Dahl et al $/ 6 /$ relative à un acier $F e ~ 460$, la ténacité $K_{l_{F}}$ a été mesurée dans la plage de vitesse de chargement $3 \times 10^{-1} \mathrm{MPa} / \mathrm{m} / \mathrm{s} \leqq \dot{K} \leqq 5 \times 10^{5} \mathrm{MP} \cdot \sqrt{\mathrm{c}} / \mathrm{s}$. Dans ce cas la courbe maitresse a été tracée en utilisant la limite d'écoulement $\sigma_{3}$ (pour $\varepsilon=0,03$ ). La valeur de la ténacité $\left(K_{I c}{ }^{\prime} \min\right.$ a été estimée à $\left(K_{1 c}{ }^{\prime}{ }_{\min }=22,7 \mathrm{MPa} \gamma^{\prime} \mathrm{et}\right.$ la contrainte de clivage $\sigma_{F}$ à $1550 \mathrm{MPa}$.

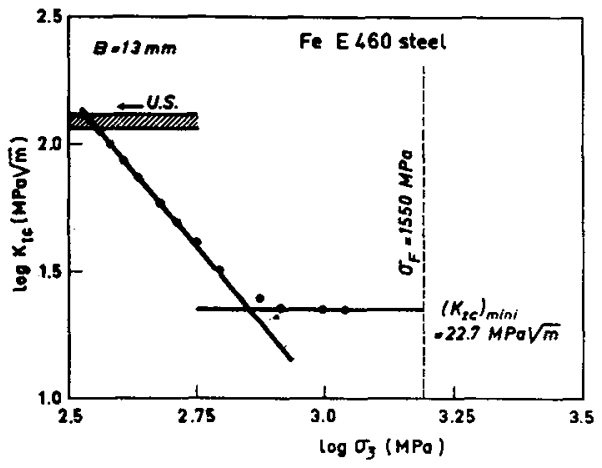

Figure 6 - Courbe maitresse pour l'acier Fe E 460 épaisseur des échantillons;

$\mathrm{B}=13 \mathrm{~mm}$. Données d'après (6)

Master plot for Fe E460 steel

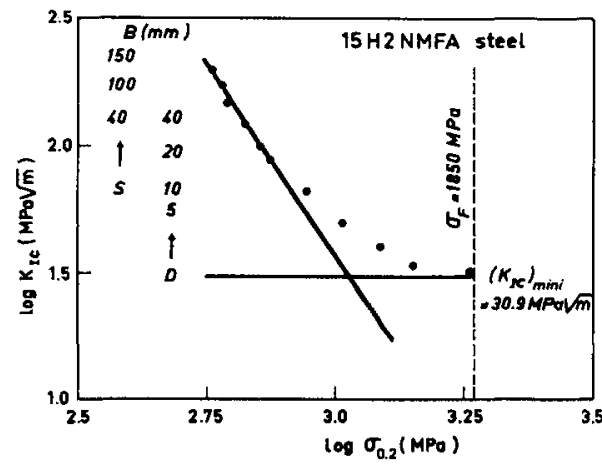

Fiqure 7 - Courbe maitresse pour l'acier 15H2NMFA (acier de cuve de réacteur).

Master plot for 15H2NMFA pres-

(German Standards), (normalized condition) sure vessel steel (Soviet Standards)

Sur la figure 7, les courtes lignes horizontales indiquent le niveau du plateau supérieur pour différentes épaisseurs $B$ dans les conditions : $S$ - quasi-statique $\dot{K}=$ $1 \times 10^{2} \mathrm{MPa} / \mathrm{m} / \mathrm{s}$ et $\mathrm{D}$ - dynamique $\dot{\mathrm{K}}=5 \times 10^{5} \mathrm{MPa} / \mathrm{m} / \mathrm{s}$, données d'après $/ 15 /$. Les données expérimentales de Krasovsky et AL /15/ obtenues sur un acier de cuve 15H2NMFA ont permis d'obtenir une courbe maitresse toute similaire avec $\left(K_{1 c}\right)_{m i n}$ $30 ; 9 \mathrm{MPa} / \mathrm{m}$ et $\sigma_{F}=1850 \mathrm{MPa}$. Bien que la corrélation dans la zone de transition, ne soit pas aussi bonne que dans le cas de l'acier $S A 508 \mathrm{Cl} .3$, les résultats sont particulièrement intéressants car ils analysent l'influence notable de l'épaisseur de l'échantillon sur le niveau du plateau supérieur, que ce soit dans des conditions 
quasi-statique (S) ou dynamique (D). Ceci montre clairement les limitations de l'essai Charpy dont les éprouvettes ont une épaisseur fixée à $10 \mathrm{~mm}$.

L'intérêt des courbes maitresses $\log k_{l c}=f\left(\log \sigma_{y}\right)$ réside dans leur utilisation comme guide dans le choix de matériaux utilisés dans différentes conditions de vitesse de chargement et de température.

\section{IV - DISCUSSION ET CONCLUSION}

La construction de la courbe maitresse repose sur une analyse de la rupture à partir d'un critère local de rupture et de la plasticité activée par la température et la vitesse de déformation 16-8/. La représentation de l'évolution de la ténacité à partir d'un seul paramètre représentant simultanément l'influence de la température et la vitesse de déformation peut se faire en utilisant le concept de température modifiée $T^{*}$. Ce type de concept a été utilisé par McGregor et Fisher $/ 21 /$ qui ont utilisé la température modifiée par la vitesse de déformation $T^{*}$

$$
T^{*}=\operatorname{Tln} \frac{\varepsilon_{0}}{\dot{\varepsilon}}
$$

$\dot{\varepsilon}_{0}$ est une constante appelée le facteur de fréquence $: 10^{8} \mathrm{~s}^{-1} \leqq \dot{\varepsilon}_{0} \leqq 10^{10} \mathrm{~s}^{-1}$. D'une façon similaire Caten et Shoemaker (22) ont utilisé une température modịfiée par la vitesse d'augmentation du facteur $d^{\prime}$ intensité de contrainte $\dot{K}$

$$
T_{K}^{*}=\operatorname{Tin} \frac{A}{\dot{K}}
$$

$A$ est une constante. En utilisant la correspondance entre la vitesse de déformation et la vitesse de chargement $\dot{K}$ on peut se placer dans des conditions équivalentes définies par la condition :

$$
T^{*}=T_{\varepsilon}^{*}=T_{K}^{*}
$$

La relation (6) permet d'obtenir la valeur de la constante A :

$$
A=\alpha \frac{E K_{l c}}{\sigma} \quad \dot{\varepsilon}_{0}
$$

Cette expression n'est toutefois pas rigoureusement constante quelle que soit la température et la vitesse de déformation mais $n^{\prime}$ en diffère que de peu car $\alpha$ et $k_{1 c}$ changent de façon opposée.

La température modifiée peut s'écrire en utilisant les logarithmes décimaux :

$$
T^{*}=\frac{T}{M}\left(C+\log \frac{\dot{\varepsilon}_{O}}{K}\right)
$$

C'est le facteur de conversion de l'équation (7) et $M=\log e$. La figure 8 montre l'évolution de la limite d'élasticité et de la ténacité en fonction de la température modifiée $\sigma_{y}\left(T^{*}\right)$ et $K_{l c}\left(T^{*}\right)$. L'élimination de $T^{*}$ permet de reconstituer la courbe maitresse de la figure 5 . 


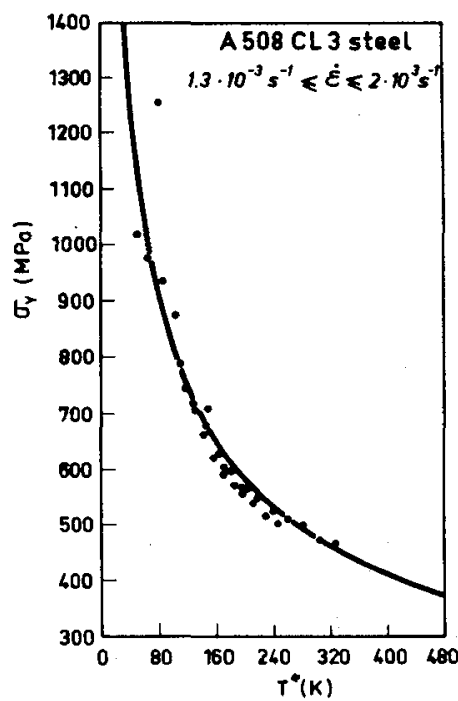

$8 \mathrm{a}$

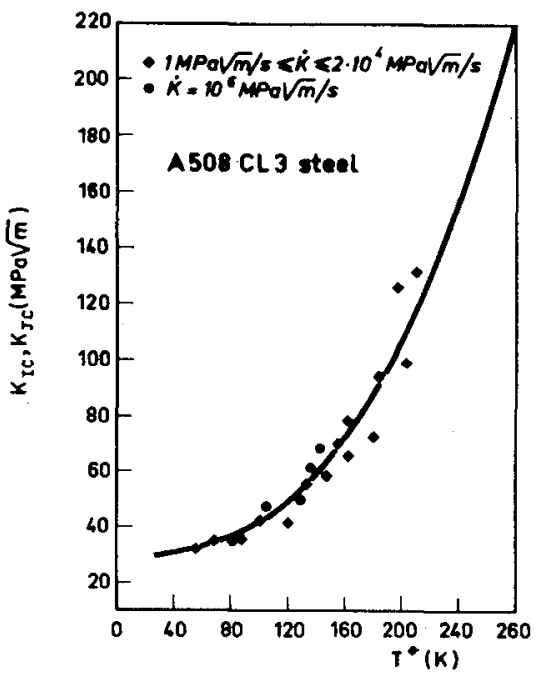

$8 \mathrm{~b}$

Figure 8 - Evolution de la limite d'élasticité $\sigma_{y}(8 a)$ et de la ténacité $K_{\text {Ic }}(8 b)$ en fonction de la température modifiée $T^{*}$ données pour l'acier SA 508cl.3.

Vield stresses $\sigma_{y}$ and fracture toughness $K_{I C}$ normalized by the modified temperature $T^{*}: a-\sigma_{y}\left(T^{*}\right) ; b-K_{I C}\left(T^{*}\right) ; a l l$ data for pressure vessel steel $A 508 \mathrm{Cl} .3$.

Les paramètres $T_{2}^{*}$ et $T_{K}^{*}$ sont basés sur la relation d'Arhenius

$$
\dot{\varepsilon}=\dot{\varepsilon}_{0} \exp \left(-\frac{\Delta G\left(\sigma^{*}\right)}{K T}\right)
$$

où $\Delta \mathrm{G}\left(\sigma^{*}\right)$ est l'énergie libre de Gibbs qui est une fonctín de la contrainte effective $\sigma^{*}$. Ces paramètres ne sont en fait qu'une façon approximative de mesurer la variation d'énergie libre car certaines conditions aux limites ne sont pas satisfaites par exemple le fait de considérer que $\sigma^{*}=\sigma$.

Des corrélations plus exactes entre la vitesse de déformation, la vitesse de chargement, la température et la ténacité sont données par ailleurs $/ 7,8,20 /$.

Cette étude montre qu'il est possible d'obtenir une bonne corrélation entre la limite d'élasticité et la ténacité en utilisant soit le concept du temps critique $t_{c}$, soit celui de la température modifiée $T^{*}$. Toutefois, l'approximation linéaire proposée dans un diagramme bilogarithmique est une approximation relativement grossière qui pourrait être affinée en considérant que la décohésion du matériau puisse être considérée elle aussi comme un phénomène activé. 


\section{REFERENCES}

/ I/ Ritchie R.0., Knott J.F. et Rice J.R., J. Mech. Phys. Solids, 21 (1973) 395.

/ 2/ Ritchie R.0., Server W.L. et Wullaert R.A., Met. Trans., 10A (1979) 1557.

/ 3/ Ritchie R.0., Server W.L. et Wullaert R.A., Proc. ICM-3, Cambridge England 3 (1979) 489.

/ 4/ Pandey R.K. et Banerjee S., Eng. Fract. Mech., 10 (1978) 817.

/ $5 /$ Pisarenko G.S., Krasovsky A.J. et Yokobori T., Reports of the Research Institute for Strength and Fracture of Materials, Tokoku University, 13 (1977) 1 .

/ 6/ Dahl W., Hesse W., Krabiell A. et Rosezin H.J., Nucl. Eng. and Design; 76 (1983) 309.

/ $7 /$ Klepaczko J.R., Proc. Conf. on Dynamic Mechanical Properties and Fracture Dynamics of Engineering Materials, Institute of Physical Metallurgy, Brno, Czechoslovakia, 3 (1983)

/ 8/ Klepaczko J.R., Theoritical and Applied Fracture Mechanics, I (1984) 181.

/ 9/ Pluvinage G. et Marandet B., Proc. Congr. ICF-6, New Dehli Iñdia (1984).

/10/ Kalthof J.F. et Shockey D.A., J. Appl. Phys., 48 (1977) 986.

/11/ Wilson M.L., Hawley R.H. et Duffy J., Eng. Fract. Mech., 13 (1980) 371.

112) Krabiell A. et Dahl W., in Advances in Fracture Research, Ed. D. François, Proc. ICF-5, Cannes France, 1 (1981) 393.

/13/ Krafft J.M., Applied Materials Res., 3 (1964) 88.

/14/ Priest A.H., Proc. Conf. on Dynamic Fracture Toughness, Weld. Inst. and ASM, Cambridge England (1976) 95.

115/ Krasovsky A.J., Kashtalyan Yu.A. et Krasiko V.N., Int. J. of Fracture 23 (1983) 297.

/16/ Hahn G.T., Hoagland R.G. et Rosenfield A.R., Met. Trans. 2A (1971) 537.

117/ Holtzman M., Vlach B. et Bilek Z., Int. J. Pres. Ves. and Piping, 9 (1981) 1.

/18/ Marandet B., Phelippeau G. et Sanz G., in Advances in Fracture Resēerch, Ed. D. François, Proc. ICF-5, Cannes France 1 (1981) 375.

/19/ Marandet M. et Pluvinage G., IRSID Final Report, PE 3729/313518, (April 1984).

120/ Garnier V., Dr. Ing. Thesis, INPL (Sept. 1984).

121/ Mc Gregor C.W. et Fisher J.C., Appl. Mech. 12(1945) A217.

122/ Corten H.T. et Shoemaker A.K., J. Basic Eng., Trans. ASME, 890 (1967) 86. 